\title{
DESENVOLVIMENTO DO SISTEMA FONOLÓGICO DE GÊMEOS MONOZIGÓTICOS COM DESVIO FONOLÓGICO: CORRELAÇÃO A FATORES GENÉTICOS E AMBIENTAIS
}

\author{
Developming the phonological systems of monozygotic \\ twins with phonological disorders: correlation with \\ genetic and environmental factors
}

\author{
Danúbia Emanuele Weber ${ }^{(1)}$, Michele Ataide Vares ${ }^{(2)}$, \\ Helena Bolli Mota ${ }^{(3)}$, Márcia Keske-Soares
}

\begin{abstract}
RESUMO
Objetivo: descrever os sistemas fonológicos de irmãos gêmeos monozigóticos com desvio fonológico e analisar a evolução da terapia fonoaudiológica após a aplicação do Modelo de Oposições Máximas Modificado a fim de investigar a influência de fatores genéticos e ambientais na instalação e manutenção das desordens fonológicas. Métodos: foram comparadas as avaliações fonológicas iniciais, os sons-alvo utilizados no planejamento terapêutico, e as avaliações fonológicas finais de cada ano de tratamento de dois sujeitos gêmeos monozigóticos do gênero masculino, com idade de seis anos e cinco meses no início da pesquisa, e com diagnóstico de desvio fonológico. Os meninos receberam atendimento de agosto de 2002 a dezembro de 2004 por meio do Modelo de Oposições Máximas Modificado. Todos os materiais foram coletados do banco de dados de um projeto desenvolvido no curso do Fonoaudiologia da Universidade Federal de Santa Maria. Resultados: grandes semelhanças foram verificadas nos sistemas fonológicos dos dois sujeitos no início, durante a evolução terapêutica e no final da terapia fonológica, apesar não terem sido utilizados necessariamente os mesmos sonsalvo no tratamento. Os meninos adquiriram os sons ausentes e parcialmente adquiridos, apresentando generalização nas diversas situações possíveis, o que comprovou a eficácia do modelo terapêutico aplicado. Conclusão: a influência genética interfere na instalação e manutenção das desordens fonológicas, porém os fatores ambientais não podem ser descartados.
\end{abstract}

DESCRITORES: Linguagem; Fala; Gêmeos; Desenvolvimento da Linguagem; Fonoaudiologia

(1) Fonoaudióloga da Escola de Educação Especial Helen Keller APAE de Três de Maio - RS; Especializanda em Fonoaudiologia pela Universidade Federal de Santa Maria - RS.

(2) Fonoaudióloga; Especializanda em Motricidade Oral pelo Cefac - Saúde e Educação - RS.

(3) Fonoaudióloga; Professora Adjunto do Curso de Fonoaudiologia e do Mestrado em Distúrbios da Comunicação da Universidade Federal de Santa Maria - RS; Doutora em Lingüística Aplicada pela Pontifícia Universidade Católica do Rio Grande do Sul.

(4) Fonoaudióloga; Professora Adjunto do Curso de Fonoaudiologia e do Mestrado em Distúrbios da Comunicação da Universidade Federal de Santa Maria - RS; Doutora em Lingüística Aplicada pela Pontifícia Universidade Católica do Rio Grande do Sul.

\section{INTRODUÇÃO}

Os irmãos, não só especificamente gêmeos, juntamente com os pais, formam o primeiro grupo social em que ocorrerá o desenvolvimento da criança. Além disso, a convivência com irmãos promove um relacionamento contínuo e longitudinal, influenciando de modo significativo o desenvolvimento de cada um. Uma concepção do irmão pode interferir no relacionamento, dificultando ou facilitando o desenvolvimento da linguagem ${ }^{1}$.

Diversos estudos, na atualidade, tomam como sujeitos, crianças gêmeas. Estas pesquisas destacam que estes sujeitos podem fornecer dados importantes em relação aos estudos da genética, que procuram identificar genes relacionados às patologias de linguagem. Estudo com famílias de crianças com desordens de linguagem evidenciou fatores genéticos relacionados às alterações ${ }^{2}$. 
Tanto na área clínica, quanto na terapêutica são inúmeros os trabalhos que estudam pares de gêmeos. Alguns destes analisam o aspecto psicológico no desenvolvimento de gêmeos, chamando a atenção para a interferência da relação existente entre esses irmãos e a possibilidade de existência de atraso no desenvolvimento da linguagem ${ }^{3}$.

Gêmeos idênticos, que se desenvolvem a partir da mesma célula-ovo e, portanto, compartilham a mesma carga genética e tornam-se mais parecidos entre si, são sujeitos a condições bastante distintas daquelas vivenciadas por gêmeos fraternos ou dizigóticos. Estes se desenvolvem a partir de duas células-ovo diferentes, tendo características físicas e psicológicas diferenciadas, o que leva cada um a ser tratado de forma mais individualizada - como "dois irmãos quaisquer", embora sem a diferença cronológica ${ }^{4-6}$. Portanto, se fica provado que a similaridade do ambiente familiar não é maior para gêmeos monozigóticos do que para os dizigóticos, então a diferença no percentual de casos em que ambos os gêmeos são afetados por desordens do desenvolvimento provém de uma estimativa da influência dos fatores genéticos causadores da disfunção ${ }^{7}$.

A maioria das dificuldades de fala e linguagem apontada em crianças gêmeas ocorre fundamentalmente em gêmeos idênticos (ou monozigóticos), o que sugere a presença de um componente genético relacionado a tais desordens ${ }^{4-7}$. Isto não significa, entretanto, que influências ambientais não sejam importantes, e não informem sobre como uma pessoa pode responder a um novo tipo de intervenção ambiental ${ }^{7}$.

O desenvolvimento da fala e da linguagem em gêmeos é atrasado, em ambos os gêneros e, em indivíduos do gênero masculino, esse atraso é de quase seis meses a mais que no gênero feminino ${ }^{6}$. Contudo, em uma pesquisa ${ }^{8}$ em que foi investigada a etiologia genética e ambiental das habilidades e desabilidades de diversos aspectos da linguagem, incluindo articulação, fonologia, gramática, vocabulário e memória verbal em uma amostra de 787 pares de gêmeos, de quatro a cinco anos de idade, de mesmo gênero e gênero oposto, os autores concluíram que as mesmas sofrem influências genéticas e ambientais similares quantitativamente e qualitativamente para meninos e meninas.

Na literatura é dado destaque ao desenvolvimento de uma linguagem própria, autônoma e particular pelos gêmeos. Entretanto, existem estudos que revelam que, na realidade, não se trata de uma linguagem autônoma, e sim uma persistência de formas imaturas de fala ${ }^{2,9}$.

Os gêmeos estudados nesta pesquisa receberam o diagnóstico de desvio fonológico. Tais alterações no desenvolvimento fonológico estão entre as mais prevalentes alterações da linguagem infantil ${ }^{10}$. As ca- racterísticas clínicas das crianças com desvio fonológico e os critérios de inclusão no distúrbio são: fala espontânea apresentando erros resultantes, principalmente, de desvios consonantais da pronúncia adulto-alvo; idade superior a quatro anos, ou seja, superior à idade na qual a fala da criança é normalmente inteligível para a maioria das pessoas; audição normal para a fala; inexistência de anormalidades anatômicas e/ou fisiológicas no mecanismo da fala; ausência de disfunção neurológica relevante; capacidades intelectuais adequadas para o desenvolvimento da linguagem falada por meio de um processo normal de socialização; e linguagem expressiva, vocabulário e extensão do enunciado, aparentemente, adequados para a idade ${ }^{11}$.

Complementando, o desvio fonológico caracteriza-se por uma alteração no desenvolvimento normal da fala em diferentes graus, com desorganização, inadaptação ou anormalidade do sistema de sons da criança em relação ao sistema padrão da comunidade lingüística a qual está inserida, tornando a fala, às vezes, ininteligível, sem que haja comprometimentos orgânicos ${ }^{12-14}$.

O tratamento dos desvios fonológicos pode ser realizado usando-se diferentes modelos de terapia fonológica, que buscarão promover uma mudança fonológica nos sistemas com desvios. Nas abordagens de tratamento voltadas para a fonologia, os sons ou as estruturas silábicas são estimulados em palavras (morfologia), em sentenças (estruturas sintáticas), em atividades que valorizam o significado dos enunciados (semântica) e seu uso em situações comunicativas reais (pragmática) ${ }^{15}$.

Existem alguns aspectos importantes a serem considerados e seguidos quando se usa uma abordagem de tratamento com base fonológica, são eles: eliminar ou minimizar os efeitos dos fatores causais, ter bem claros os objetivos do tratamento e os procedimentos necessários para atingi-los, promover generalização incluindo atividades de transferência e de manutenção das produções aprendidas no tratamento, avaliar continuamente o andamento da terapia, fazer do paciente um participante ativo do processo terapêutico, envolver pais, irmãos e professores no tratamento, manter um acompanhamento do paciente durante um período de tempo (de três a seis meses) após sua alta da terapia ${ }^{15}$.

O modelo terapêutico de Oposições Máximas ${ }^{16,17}$ envolve pares de duas palavras que se diferenciam em apenas um fonema. Se estes fonemas diferem em apenas um traço distintivo, formam as oposições mínimas, e se diferem em vários traços constituem oposições máximas.

O objetivo da utilização de contrastes de pares mínimos no tratamento de crianças com desvio fonológico é fazê-las distinguir pares de sílabas ou palavras (por meio de discriminação, imitação, e/ou produção espon- 
tânea), que são únicos em suas características ou dimensões. Por meio do tratamento com pares mínimos, uma criança é ensinada que diferentes sons assinalam diferentes significados. $O$ tratamento que utiliza contraste de pares mínimos reduz a homonímia na produção da criança pelo contraste realizado entre os sonsalvo errados ou substituídos ${ }^{16,17}$.

As palavras de um par mínimo podem distinguirse também quanto à natureza das distinções dos traços e quanto à relação dos fonemas contrastantes com a gramática da criança antes do tratamento ${ }^{16,17}$.

Quanto à natureza das distinções de traços, os fonemas podem diferir por traços de classe principal ou não-principal. Os primeiros descrevem e distinguem as grandes classes de sons, como vogais, semivogais e consoantes, obstruintes, líquidas e nasais, referindo-se a diferenças que envolvem os traços que fazem parte do nó de raiz dos fonemas: [soante], [aproximante] e [vocóide]. Os segundos referem-se a todas as outras diferenças de traços de ponto, modo e sonoridade entre os fonemas ${ }^{16,17}$.

Quanto à relação dos fonemas contrastantes com a gramática da criança antes do tratamento, os fonemas podem ser fonologicamente conhecidos ou desconhecidos. Em geral, o som-alvo de tratamento deve ser desconhecido, ou seja, não funcional no inventário fonêmico da criança. $O$ fonema contrastante pode ser conhecido ou desconhecido ${ }^{15}$.

O Modelo de Oposições Máximas ${ }^{16,17}$ pode apresentar três níveis de generalização conforme os pares de fonemas contrastados. O primeiro deles envolve dois fonemas novos e oposição de classe principal; o segundo envolve ou dois fonemas novos e oposição de classe não-principal, ou um fonema novo e oposição de classe principal; e o terceiro e último, que tende a promover menor generalização, engloba um fonema novo e oposição de classe não-principal ${ }^{18}$.

Este modelo terapêutico, apesar de pouco relatado bibliograficamente, foi rápido e eficaz na terapia de fala de crianças com desvio fonológico ${ }^{19,20}$.

No tratamento fonológico dos gêmeos desta pesquisa, foi utilizado o modelo de Oposições Máximas Modificado ${ }^{21}$. Este modelo sugere algumas modificações no Modelo de Oposições Máximas, sendo que sua aplicação foi testada, com sucesso, em pacientes com desvios fonológicos falantes do português brasileiro. Foram introduzidos ao modelo antigo, procedimentos relacionados à escolha dos sons-alvo, como o controle do ambiente fonético e a utilização de palavras com significado; procedimentos referentes à terapia fonológica propriamente dita, como o bombardeio auditivo e a prática de orientações aos pais e, também, procedimentos relacionados à estrutura da sessão terapêutica, como avaliações fonológicas periódicas ${ }^{21}$.

O objetivo do Modelo de Oposições Máximas Modificado ${ }^{21}$ é o mesmo do modelo original, ou seja, promover a reorganização do sistema fonológico da criança por meio da percepção auditiva, imitação da produção e produção espontânea dos sons-alvo, visando a generalização e a melhora na inteligibilidade da fala da criança.

Para a escolha dos alvos de tratamento, os seguintes procedimentos são sugeridos ${ }^{15,21}$ : 1) coleta da fala da criança com gravação da mesma, por meio da nomeação e conversa espontânea, utilizando um instrumento que contenha palavras com todos os sons do português, nas diferentes posições em que estes podem acontecer; 2) transcrição fonética dos dados coletados e análise fonológica por meio da análise contrastiva e por traços distintivos, obtendo-se, então, o sistema fonológico da criança, e sendo possível determinar os fonemas que estão adquiridos quais não estão e os traços distintivos que a criança apresenta dificuldades, segundo critérios de análise fonológica; 3) escolha dos fonemas-alvo baseandose na hierarquia de mudanças fonológicas ${ }^{17}$, dando preferência para o formato que leva a maior mudança fonológica, como também selecionar alvos que contenham os traços distintivos para os quais a criança apresenta dificuldade; 4) seleção dos pares mínimos, podendo ser escolhido de dois a oito pares, nos quais os fonemas-alvo devem estar sempre na mesma posição na palavra.

É ressaltado na literatura ${ }^{20}$ que o conhecimento e a utilização de hierarquias implicacionais podem prever as generalizações estruturais na terapia dos sujeitos com alterações de fala.

Para os procedimentos de terapia, sugere-se ${ }^{15,21}$ : 1) iniciar com a determinação de uma linha de base para cada som-problema. Essa linha é uma sondagem realizada antes de se iniciar o tratamento fonológico. Para realizá-la deve-se verificar quais os fonemas ausentes e parcialmente adquiridos no sistema fonológico da criança nas diferentes posições. Cada fonema não adquirido deve ser testado selecionando-se seis palavras que contenham esse fonema e que possam ser representadas por figuras, sendo que estas devem ser mostradas à criança e ela deverá reproduzir os seus nomes. Em cada palavra selecionada para essa testagem, o único fonema ausente ou parcialmente adquirido deve ser o testado. A linha de base serve de parâmetro inicial de comparação dos progressos do tratamento e as generalizações ocorridas no seu andamento. 2) Realizar cinco sessões de estimulação com os pares mínimos selecionados. 3) Na sexta sessão, realizar a sondagem da generalização, que deve ser feita do mesmo modo da linha de base. 4) Repetir mais cinco sessões com os mesmo pares mínimos. 5) Novamente, na sexta sessão, realizar a sondagem da generalização. 6) Continuar a terapia realizando sempre cinco sessões de estimulação e na sexta sessão, uma sondagem de generalização. 7) Após 20 sessões de 
terapia (sem contar as de sondagem), realizar nova avaliação geral do sistema fonológico da criança.

Em relação à estruturação da sessão de terapia, é recomendado que ${ }^{15,21}:$ 1) Cada sessão deve ser iniciada com a leitura para a criança de um bombardeio auditivo, que consiste em uma lista de 16 palavras, das quais oito devem conter um dos fonemas-alvo e as oito restantes o outro fonema-alvo. 2) A estimulação da produção correta dos pares mínimos é realizada em duas etapas: uma de imitação e a outra de produção espontânea. Na imitação a criança deve produzir os fonemas-alvo após o modelo da terapeuta; na produção espontânea os alvos devem ser produzidos sem o modelo imediato. No final da sessão, deve-se calcular a porcentagem de produções corretas realizadas pela criança. Na fase de imitação a criança deve atingir um percentual de produções corretas de $80 \%$ ou mais. Nesse caso, na sessão seguinte, deve-se passar para a fase de produção espontânea. 3) No final da sessão ler novamente a lista de palavras do bombardeio auditivo. 4) Realizar orientações aos pais ou responsáveis: a lista do bombardeio auditivo deve ser entregue a eles para que seja lida uma vez por dia para a criança; devem ser entregues cópias das figuras-alvo trabaIhadas em terapia e sugerir atividades para serem realizadas em casa.

A escassez de pesquisas nacionais sobre desvio fonológico em gêmeos monozigóticos foi fator motivador para este estudo, tendo em vista a importância dessas para a ampliação dos conhecimentos da comunidade científica e também para o fornecimento de subsídios a futuras pesquisas.

Assim, o presente artigo teve por objetivo descrever os sistemas fonológicos de irmãos gêmeos monozigóticos com desvio fonológico, analisar aspectos da evolução da terapia fonoaudiológica dessas crianças após a aplicação do Modelo de Oposições Máximas Modificado ${ }^{21}$, além de verificar sua eficácia.

\section{MÉTODOS}

Esta pesquisa foi delineada como um estudo longitudinal, exploratório, descritivo e qualitativo de dados obtidos em prontuários do projeto "Estudo comparativo da generalização em três modelos de terapia fonológica em crianças com diferentes graus de severidade do desvio fonológico", desenvolvido no Centro de Estudos de Linguagem e Fala (CELF) do Serviço de Atendimento Fonoaudiológico (SAF) da Universidade Federal de Santa Maria (UFSM).

A autorização específica dos pais e/ou responsáveis para participação dos sujeitos não foi solicitada para esta pesquisa, uma vez que a mesma envolveu investigação de dados em prontuários do projeto já referido, o qual empregou Consentimento Livre e Esclarecido próprio.
Os sujeitos deste trabalho eram gêmeos monozigóticos do gênero masculino, idade de seis anos e cinco meses ao iniciarem os procedimentos avaliativos no SAF e apresentavam queixa de alterações na fala. Todos os dados necessários e convenientes a esta pesquisa estavam disponíveis nos arquivos do projeto mencionado.

Os sujeitos foram avaliados, primeiramente, por meio de triagem fonoaudiológica realizada no próprio SAF, na qual foi diagnosticado desvio fonológico e feito o encaminhamento para o CELF, onde ambos foram submetidos a novas avaliações, estas realizadas por terapeutas estagiárias do último ano de graduação em Fonoaudiologia da UFSM, sob orientação das professoras coordenadoras do projeto. Tal diagnóstico foi confirmado por meio de avaliações fonoaudiológicas, a saber, Avaliação Fonológica da Criança-AFC ${ }^{22}$; Avaliação da Linguagem Expressiva e Compreensiva, efetuada de modo informal, observando-se os aspectos compreensivo e expressivo, bem como a capacidade de execução de ordens, organização e expressão da estrutura gramatical, e uso de enunciados no contexto comunicativo; Exame Articulatório; Avaliação do Sistema Estomatognático ${ }^{23}$, verificando as condições anatomofisiológicas e o funcionamento dos órgãos fonoarticulatórios; Avaliação da Percepção Fonêmica; Avaliação Psicomotora; Avaliação da Consciência Fonológica ${ }^{24}$; Avaliação da Memória de Trabalho;Avaliação Simplificada do Processamento Auditivo ${ }^{25}$; e Avaliação do Vocabulário; e exames complementares, como Avaliação Otorrinolaringológica e Avaliação Neurológica, executadas por médicos professores e integrantes da equipe multidisciplinar do serviço, Avaliação Audiológica e Avaliação do Processamento Auditivo, também realizadas no SAF.

O tratamento foi iniciado em agosto de 2002 e finalizado em dezembro de 2004, por meio do Modelo Oposições Máximas Modificado ${ }^{21}$. Os sujeitos eram atendidos individualmente, por terapeutas estagiárias diferentes. Iniciou-se com a coleta dos dados da fala, mediante gravação da fala espontânea e aplicação do instrumento AFC ${ }^{22}$, seguido da transcrição e da análise fonológica por meio da análise contrastiva. Então, foram determinados os fonemas que estavam adquiridos e os que não estavam, e, a partir disto, foram delimitados os sons-alvo para o tratamento, baseandose na hierarquia de mudanças fonológicas ${ }^{17}$. Também foram estabelecidas as respectivas palavras-alvo (com os fonemas-alvo na mesma posição na palavra) e as do bombardeio auditivo, composto de 16 palavras, sendo oito com cada fonema-alvo, também, na mesma posição na palavra.

Foi elaborada uma linha de base ${ }^{15-21}$, na qual eram selecionadas cinco figuras representando palavras que contivessem cada um dos fonemas não-adquiridos, em cada uma das posições. Eram realizadas 
cinco sessões e, na sexta sessão, uma sondagem nos moldes da linha de base. Após 20 sessões de terapia, era realizada uma nova AFC ${ }^{22}$. Cada sessão começava e era finalizada com a leitura do bombardeio auditivo e a estimulação da produção correta dos pares mínimos era realizada, primeiramente, por meio da imitação e, posteriormente, da produção espontânea.

Para esta pesquisa foram alçados na literatura conceitos, afirmações e achados de outros estudos referentes a: gêmeos, aspectos genéticos relacionados à aquisição e ao desenvolvimento da linguagem e da fala, características dos desvios fonológicos, tratamento para desvios fonológicos, e modelo de oposições máximas modificado. Com esta finalidade, utilizou-se livros, periódicos científicos, anais de congressos e demais eventos científicos, além de publicações veiculadas pela internet.

Assim, após a revisão de literatura, que serviu como base para a discussão, foram observados e comparados, a AFC inicial, os sons-alvo utilizados no planejamento terapêutico, e as AFC finais dos sujeitos em cada ano de tratamento, estando os dados relatados especificadamente na Figura 1.

O projeto mencionado, que serviu como base para esta pesquisa, tem registro no Gabinete de Projetos do Centro de Ciências da Saúde da Universidade Federal de Santa Maria (GAP/CCS) sob nำ12650, e no Comitê de Ética em Pesquisa da instituição de origem (CEP/UFSM) sob nํㅜ 046/02.

\section{RESULTADOS}

O Sujeito 1 (S1) chegou ao SAF, acompanhado pelos pais, com queixa de dificuldades na fala, tendo sido encaminhado pela professora da creche. Não há relatos de intercorrências quanto à gestação, parto e desenvolvimento neuropsicomotor, bem como de atraso no início da fala.

$\mathrm{Na}$ AFC inicial, realizada em agosto de 2002, o $\mathrm{S} 1$, em onset, possuía adquiridas as plosivas /p/, $/ \mathrm{t} / \mathrm{e} / \mathrm{k} /$, as fricativas $/ \mathrm{f} / \mathrm{e} / \mathrm{s} /$, as nasais $/ \mathrm{m} / \mathrm{h} / \mathrm{n} / \mathrm{e}$ $/ \mathrm{n} /$, e as líquidas laterais $/ / / \mathrm{e} / \mathcal{K} /$. A plosiva $/ \mathrm{t} / \mathrm{e}$ a fricativa /f/ estavam parcialmente adquiridas. Estavam ausentes as plosivas /b/, /d/ e /g/, as fricativas /z/, / / / e / 3 /, as líquidas não-laterais / $/$ e / R/, bem como os alofones [t $\int$ ] e [dz]. Em coda, a fricativa /s/ estava parcialmente adquirida e a líquida não-lateral / $r /$ estava ausente. Os encontros consonantais (EC) estavam reduzidos a uma só consoante, não havendo a produção da estrutura silábica consoante-consoante-vogal (CCV).

Assim, foram determinados os sons-alvo para o tratamento fonológico do ano de 2003, sendo os mesmos: / $/ \mathrm{x} / \mathrm{r} /$ em OM (onset medial), $/ \mathrm{z} / \mathrm{x} / \mathrm{r} /$ em OM, / $/$ x /r/ em OM, e /d/ x/3/ em Ol (onset inicial), e OM. A escolha de tais sons era baseada no sistema fonológico obtido por meio das reavaliações, realizadas a cada 20 sessões de terapia, bem como na hierarquia de mudança fonológica ${ }^{12}$, selecionando-se sons que poderiam provocar maiores ou menores mudanças fonológicas, a fim de se obter generalizações.

Ao término do ano de 2003, na AFC final, o S1 possuía adquiridas as plosivas /p/, /t/ e /k/, as fricativas $/ \mathrm{f} / \mathrm{e} / \mathrm{s} /$, as nasais $/ \mathrm{m} /, \mathrm{n} / \mathrm{e} / \mathrm{j} /$, e as líquidas ///, /א/, /r/ e /R/. As plosivas /b/, /d/ e /g/, e as fricativas $/ \mathrm{v} / \mathrm{e} / \mathrm{z} /$ estavam parcialmente adquiridas. Estavam ausentes as fricativas / $\mathrm{/} \mathrm{e} \mathrm{/3/,} \mathrm{e} \mathrm{os}$ alofones [ $\mathrm{t} \int$ ] e $\left[\mathrm{d}_{3}\right]$. Em coda, a fricativa $/ \mathrm{s} / \mathrm{e}$ a líquida não-lateral /r/ estavam adquiridas. Os EC estavam parcialmente adquiridos, em sua maioria.

Em 2004, foram trabalhados os pares: /z/ x / $\mathcal{K} /$ em OM e /g/ x /v/ em OM.

A fim de concluir os atendimentos do ano de 2004, o paciente foi reavaliado, constatando-se que o mesmo adquiriu todos os fonemas e alofones, assim como os EC, exceto a fricativa / 3/, que se encontrava parcialmente adquirida. Portanto, o sujeito foi desvinculado do projeto do qual participava.

O Sujeito 2 (S2) chegou ao SAF com as mesmas queixas e relatos do $S 1$.

$\mathrm{Na}$ AFC inicial, o S2, em onset, possuía adquiridas as plosivas /p/ e /k/, a fricativa /f/, as nasais $/ \mathrm{m} /$, $/ \mathrm{n} / \mathrm{e} / \mathrm{j} /$, e as líquidas laterais // e / $\mathcal{\mathcal { L }} /$. As fricativas /s/ e /z/, e a líquida não-lateral /R/ estavam parcialmente adquiridas. Estavam ausentes as plosivas $/ \mathrm{b} /$, /t/, /d/ e /g/, as fricativas /v/, / / / e /3/, a líquida nãolaterais $/ \mathrm{r} /$, bem como os alofones [ $\mathrm{t} \int$ ] e [ $\left.\mathrm{d}_{3}\right]$. Em coda, a fricativa /s/ e a líquida não-lateral/r/ estavam ausentes. Os EC estavam reduzidos a uma só consoante.

A partir de então, para a terapia até o final do ano de 2003, foram selecionados os seguintes sons-alvo em pares: /g/ x /z/ em OI. /v/ x /R/ em OI, /r/ x /t/ em $\mathrm{OM}, / 3 / \mathrm{x} / \mathrm{r} / \mathrm{em} \mathrm{OM}, / 3 / \mathrm{x} / \mathrm{r} / \mathrm{em} \mathrm{OM}, / \mathrm{t} / \mathrm{x} / 3 / \mathrm{em}$ Ol e OM, e /d/ x/3/ em Ol e OM.

Ao final de 2003 foi realizada uma nova AFC, com os seguintes achados: estavam adquiridas, em onset, as plosivas /p/, /t/ e /k/, as fricativas /f/ e $/ \mathrm{s} /$, as nasais $/ \mathrm{m} /, / \mathrm{n} / \mathrm{e} / \mathrm{j} /$, e as líquidas $/ / /, / \mathcal{K} /$, /r/ e /R/. Estavam ausentes as plosivas /b/, /d/ e / $\mathrm{g} /$, as fricativas $/ \mathrm{v} /, / \mathrm{z} /, / \mathrm{J} / \mathrm{e} / 3 /$, e os alofones [t $\left.\int\right]$ e [dz]. Em coda, a fricativa /s/ e a líquida não-lateral /r/ estavam adquiridas. Os EC estavam parcialmente adquiridos.

Para o ano seguinte, novos sons-alvo foram selecionados, sendo eles: / $3 / \mathrm{x} / \mathrm{b} / \mathrm{em} \mathrm{Ol,} / \mathrm{g} / \mathrm{x} / \mathrm{v} / \mathrm{em}$ $\mathrm{OM}$, e /t/ x /d/ em OI.

Na reavaliação final de 2004, observou-se que todos os fonemas e alofones haviam sido adquiridos, e, também, houve generalização para estruturas mais complexas do tipo CCV. Dessa forma, o sujeito foi desvinculado do projeto do qual participava. 


\begin{tabular}{|c|c|c|c|c|c|c|c|}
\hline Posição & & \multicolumn{2}{|c|}{ AFC I } & \multicolumn{2}{|c|}{ AFC F 2003} & \multicolumn{2}{|c|}{ AFC F 2004} \\
\hline Onset & & S1 & S2 & S1 & S2 & S1 & S2 \\
\hline & $\mathrm{SA}$ & $\begin{array}{c}/ \mathrm{p} /, / \mathrm{k} /, / \mathrm{f} /, / \mathrm{s} / \\
/ \mathrm{m} /, / \mathrm{n} /, / \mathrm{n} /, / \mathrm{l} / \\
/ \mathrm{S} /\end{array}$ & $\begin{array}{l}/ \mathrm{p} /, / \mathrm{k} /, / \mathrm{f} /, / \mathrm{m} / \\
/ \mathrm{n} /, / \mathrm{N} /, / / /, / \mathcal{S} /\end{array}$ & $\begin{array}{l}/ \mathrm{p} /, / \mathrm{t} /, / \mathrm{k} /, / \mathrm{f} / \\
/ \mathrm{s} /, / \mathrm{m} /, / \mathrm{n} /, / \mathrm{N} / \\
/ \mathrm{I} /, / \mathcal{N} /, / \mathrm{r} /, / \mathrm{R} /\end{array}$ & $\begin{array}{c}/ \mathrm{p} /, / \mathrm{t} /, / \mathrm{k} /, / \mathrm{f} / \\
/ \mathrm{s} /, / \mathrm{m} /, / \mathrm{n} /, / \mathrm{n} / \\
/ \mathrm{l} /, / \mathrm{S} /, / \mathrm{r} /, / \mathrm{R} /\end{array}$ & $\begin{array}{l}/ \mathrm{p} /, / \mathrm{b} /, / \mathrm{tt} /, / \mathrm{d} /, \\
/ \mathrm{k} /, / \mathrm{g} /, / \mathrm{ff} /, / \mathrm{v} /, \\
/ \mathrm{s} /, / \mathrm{z} /, / \int I, / \mathrm{m} /, \\
/ \mathrm{n} /, / \mathrm{J} /, / \mathrm{l} /, / \mathcal{S} / \\
/ \mathrm{r} /, / \mathrm{R} /,\left[\mathrm{t} \int\right],\left[\mathrm{d}_{3}\right]\end{array}$ & $\begin{array}{c}/ \mathrm{p} /, / \mathrm{b} /, \mid \mathrm{t} /, / \mathrm{d} /, \\
/ \mathrm{k} /, / \mathrm{g} /, / \mathrm{f} /, / \mathrm{v} /, \\
/ \mathrm{s} /, \mid \mathrm{z} /, / \mathrm{s} /, / 3 /, \\
/ \mathrm{m} /, / \mathrm{n} /, / \mathrm{d} /, / / /, \\
/ \mathcal{S} /, / \mathrm{r} /, / \mathrm{R} /,\left[\mathrm{t} \int\right], \\
{\left[\mathrm{d}_{3}\right]}\end{array}$ \\
\hline & SPA & $/ \mathrm{t} / \mathrm{I} / \mathrm{s}$ & $|\mathrm{s} /,| \mathrm{z} \mid, / \mathrm{R} /$ & $\begin{array}{c}/ \mathrm{b} /, / \mathbf{d} /, / \mathrm{g} /, / \mathrm{v} / \\
\mid \mathrm{z} /\end{array}$ & & 131 & \\
\hline & SNA & $\begin{array}{c}/ \mathrm{b} /, / \mathrm{d} /, / \mathrm{g} /, / \mathrm{z} /, \\
/ \int /, / 3 /, / \mathrm{r} /, / \mathrm{R} /, \\
{\left[\mathrm{t} \int\right],\left[\mathrm{d}_{3}\right]}\end{array}$ & $\begin{array}{c}/ \mathrm{b} /, / \mathrm{t} /, / \mathrm{d} /, / \mathrm{g} /, \\
/ \mathrm{v} /, / \int /, / 3 /, / \mathrm{r} /, \\
{\left[\mathrm{t} \int\right],\left[\mathrm{d}_{3}\right]}\end{array}$ & $/ \int /, / 3 /,\left[\mathrm{t} \int\right],\left[\mathrm{d}_{3}\right]$ & $\begin{array}{c}/ \mathrm{b} /, / \mathrm{d} /, / \mathrm{g} /, / \mathrm{v} / \\
/ \mathrm{z} /, / \int /, / 3 /,\left[\mathrm{t} \int\right] \\
{\left[\mathrm{d}_{3}\right]}\end{array}$ & & \\
\hline Coda & & S1 & S2 & S1 & S2 & S1 & S2 \\
\hline & SA & & & $|\mathbf{s} /, / \mathbf{r}|$ & $|\mathbf{s}|,|\mathbf{r}|$ & $|\mathrm{s} /,| \mathrm{r} /$ & $\mid \mathrm{s} /, / \mathrm{r} /$ \\
\hline & SPA & $/ \mathrm{s} /$ & & & & & \\
\hline & SNA & $\mid r /$ & $|\mathrm{s} /, / \mathrm{r}|$ & & & & \\
\hline
\end{tabular}

AFC - Avaliação fonológica da criança

AFC I: AFC inicial. AFC F 2003: AFC final de 2003. AFC F 2004: AFC final de 2004. SA: sons adquiridos. SPA: sons parcialmente adquiridos. SNA: sons não adquiridos. S1: sujeito 1. S2: sujeito 2.

\section{Figura 1 - Mudanças fonológicas ocorridas em S1 e S2 durante o tratamento fonológico}

\section{DISCUSSÃo}

A discussão deste trabalho enfocará os aspectos comuns da fala dos sujeitos descritos, desde a AFC inicial até a AFC final, comentando a evolução terapêutica por meio do Modelo de Oposições Máximas Modificado ${ }^{21}$, e correlacionando os achados à literatura que faz referência à influência genética e ambiental na fala de gêmeos.

Os sujeitos S1 e S2 chegaram ao serviço tendo adquiridos, em seus sistemas fonológicos, na posição de onset, os seguintes sons em comum: /p/, / / , $/ \mathrm{f} /, / \mathrm{m} /, / \mathrm{n} /, / \mathrm{j} /, / / / \mathrm{e} / \mathrm{K} /$. Os sons $/ \mathrm{b} /, / \mathrm{d} /, / \mathrm{g} /, / \mathrm{s} /$, $/ 3 /, / r /,\left[t \int\right]$ e $\left[d_{3}\right]$, na mesma posição, estavam ausentes nos sistemas fonológicos de ambos os meninos. Em coda, /r/ não estava adquirido pelos dois sujeitos. Como se pode observar havia semelhança entre os sistemas fonológicos dos gêmeos antes da intervenção, indo ao encontro dos achados da literatura no que diz respeito às similaridades no que tange à fala e à linguagem de gêmeos monozigóticos, 0 que pode ser determinado tanto por influência ambiental, quanto genética ${ }^{4-7}$.

O S1 apresentava, em onset, /s/ adquirido, /t/ e / $\mathrm{v} /$ parcialmente adquiridos, e /R/e/z/, ausentes; em coda, /s/ estava parcialmente adquirido. O S2 tinha /s/, /z/ e /R/ parcialmente adquiridos, e /t/ e / / ausentes, em onset, e /s/, em coda, ausente.

No ano de 2003, no processo terapêutico do S1, com o modelo descrito, foram utilizados, em pares, os fonemas-alvo $/ \mathrm{d} / \mathrm{,} / \mathrm{z} /, / \mathrm{S} /, / 3 / \mathrm{e} / \mathrm{r} /$, e o mesmo adquiriu os fonemas /t/, /r/ e /R/, e os fonemas /b/, /d/, /g/ e /z/ tornaram-se parcialmente adquiridos. Já, com o S2, além daqueles fonemas-alvo, foram utilizados ainda /t/, /g/, / / / e /R/, e, neste ano, o S2 adquiriu, também em onset, /t/, /s/, /r/ e /R/. Ambos adquiriram, em coda, os fonemas $/ \mathrm{s} / \mathrm{e} / \mathrm{r} /$.

Na reavaliação do final de 2003, havia em comum entre os meninos, em onset, /p/, /t/, / / /, /f/, /s/, /m/, /n/, $/ \mathrm{n} /, / / /, / \mathcal{K} /, / \mathrm{r} / \mathrm{e} / \mathrm{R} /$ adquiridos, e $/ \mathrm{S} /, / 3 /$, [t $\mathrm{s}] \mathrm{e}$ [d] não adquiridos, o que demonstra que neste ano $/ \mathrm{t} /, / \mathrm{r} / \mathrm{e} / \mathrm{R} /$ foram os fonemas adquiridos igualmente pelos dois sujeitos; além de /s/e/r/na posição de coda. Nos dois casos, pôde-se perceber que determinados sons foram adquiridos, embora não tenham sido trabaIhados diretamente em terapia, o que nos remete à generalização, tanto para itens não tratados, quanto para outra posição na palavra, para a mesma classe de sons, para outras classes de sons, e para outras unidades lingüísticas, o que concorda com outro estudo ${ }^{26} \mathrm{em}$ 
que, utilizando-se o Modelo de Oposições Máximas no tratamento de crianças com desvio fonológico, obtevese variados tipos de generalizações estruturais. No caso de S1, houve generalização para /t/ e /R/, em onset, e/s/ em coda, e, no S2 as generalizações ocorreram para/s/ em onsete coda.

Para a terapia fonológica aplicada em 2004, foram selecionados como alvos para o $\mathrm{S} 1$ /g/, /V/, /z/ e / $\mathcal{S}$, e para o S2, /b/, /t/, /d/, /g/, /v/ e / 3/. Ao final do ano, após a AFC final, observou-se que todos os sons foram adquiridos, exceto / 3/ para o S1, que se tornou parcialmente adquirido. Os fonemas em comum adquiridos pelos gêmeos, neste ano, foram:/b/, /d/, /g/, /v/, /z/, / $\int /$ e os alofones [t $\left.\int\right]$ e [dz]. Novamente, ocorreram generalizações, sendo as mesmas para o $\mathrm{S} 1$ :

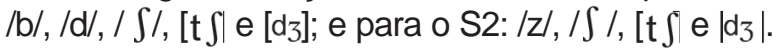

Verifica-se que houve momentos da terapia em que não mais havia como contrastar fonemas em oposições máximas, devido ao fato de os pacientes já terem adquiridos grande parte dos fonemas. Dessa forma, optou-se por contrastar fonemas em oposições mínimas ${ }^{17}$.

Ao final de 2004 os objetivos da terapia haviam sido alcançados, ou seja, ocorreram generalizações e os sistemas fonológicos foram reorganizados, corroborando novamente à eficácia do Modelo de Oposições Máximas, conforme já relatado por algumas autoras ${ }^{21}$.

No decorrer dos dois anos de terapia fonológica, foi notada uma evolução semelhante no desenvolvimento do sistema fonológico dos gêmeos, mesmo não tendo sido aplicado um planejamento igual para ambos, visto que os pares escolhidos não eram utilizados ao mesmo tempo com os dois meninos. Todavia, esta afirmação vai de encontro a uma pesquisa ${ }^{1}$ em que um casal de gêmeos que apesar de terem sido submetidos ao mesmo número de ses- sões fonoaudiológicas, planejadas em conjunto pelas terapeutas, sob as mesmas condições e orientações, não necessariamente seguiram o mesmo caminho de desenvolvimento.

Conforme estudo, aproximadamente $90 \%$ dos pares de gêmeos monozigóticos apresentam uma história de desenvolvimento semelhante no que tange à fala e à linguagem, comparativamente à proporção de $40 \%$ entre os gêmeos dizigóticos, atribuindo, assim a maior semelhança entre os primeiros à herança genética compartilhada. Também há indicações de que $80 \%$ da variação no desenvolvimento da linguagem em gêmeos são devido a fatores hereditários e somente cerca de $10 \%$ a fatores ambientais ${ }^{6}$.

Este relato de caso vem reforçar o que pesquisas ${ }^{4-7}$ na área mencionam a respeito da contribuição genética para a manifestação de alterações de fala e linguagem em crianças gemelares, predominantemente em gêmeos monozigóticos e do gênero masculino, contudo, sem descartar a influência do ambiente, já que os mesmos podem adotar um ao outro como modelo, gerando assim um reforço, ora positivo, ora negativo.

\section{CONCLUSÃO}

Após a realização deste estudo, foi possível constatar que houve similaridade entre os sistemas fonológicos iniciais dos gêmeos anteriormente à intervenção; a evolução terapêutica foi semelhante, pois os irmãos adquirem comumente vários fonemas; foi necessário o mesmo tempo de terapia para que ambos os meninos completassem seus sistemas fonológicos; a eficácia do modelo terapêutico aplicado está comprovada, já que, além dos sons trabalhados, proporciona a generalização nas diversas situações possíveis.

\section{ABSTRACT}

Purpose: to describe the phonological systems of monozygotic twins both having phonological disorders and to analyze the gradual development of the speech therapy after applying Modified Maximal Opposition Model in order to investigate the influence of genetic and environmental factors on the outset and continuity (maintenance) of phonological disorders. Methods: the initial phonological assessments were compared as well as the target-sounds used in therapeutic plan, and the final phonological assessments at the end of each year of treatment for both monozygotic male twins who were six years and five months of age at the beginning of the research, and diagnosed as having phonological disorders. The boys were treated from August 2002 to December 2004 according to the Modified Maximal Opposition Model. All the material was obtained from the database of a project developed in the speech/language clinic of the Federal University of Santa Maria. Results: great similarities were found in the phonological systems of both subjects in the beginning, during the therapeutic evolution and at the end of the phonological therapy, even though the target sounds were not necessarily the same throughout therapy. The boys acquired the absent and partially acquired sounds, presenting generalization in the various possible situations, confirming the efficiency of the therapeutic model employed. Conclusion: the genetic influence induces the onset and continuity of phonological disorders; nevertheless environmental factors cannot be disregarded.

KEYWORDS: Language; Speech; Twins; Language Development; Speech, Language and Hearing Sciences 


\section{REFERÊNCIAS}

1. Monteiro MIB, Bagarollo MF. Os irmãos e o desenvolvimento da linguagem de sujeitos deficientes mentais. Disturb Comum. 2004; 16(2):253-9.

2. Befi-Lopes DM, Morselli AA, Araújo K. Intervenção fonoaudiológica em gêmeos com alteração de linguagem: relato de caso. J Bras Fonoaudiol. 2001; 2(7):116-25.

3. Limongi SCO, Andrade RS, Lima FAGF, Alabarse VM, Perez VM. Processo terapêutico fonoaudiológico realizado com um par de gêmeos portadores de síndrome de Down. Pró-Fono R Atual Cient. 2002; 12(1):24-33.

4. Bishop DV. The role of genes in the etiology of specific language impairment. J Commun Disord. 2002; 35(4):311-28.

5. Barbetta NL, Panhoca I. Gêmeos idênticos no grupo terapêutico-fonoaudiológico: a construção da linguagem e subjetividade. Pró-Fono R Atual Cient. 2003; 15(2):139-48.

6. Lewis BA, Thompson LA. A study of developmental speech and language disorders in twins. J Speech Hear Res. 1992; 35(5):1086-94.

7. Bishop DVM. The biological basis of specific language impairment. In: Fletcher P, Hall D. Specific speech \& language disorders in children. California: Singular Publishing Group; 1992. p. 2-17.

8. Kovas Y, Hayiou-Thomas ME, Oliver B, Dale PS, Bishop DV, Plomin R. Genetic influences in different aspects of language development: the etiology of language skills in 4.5-year-old twins. Child Dev. 2005; 76(3):632-51.

9. Bishop DV, Bishop SJ. "Twin language": risk factor for language impairment? J Speech Lang Hear Res. 1998; 41(1):150-60.

10. Wertzner HF, Ramos AC, Amaro L. Índices fonológicos aplicados ao desenvolvimento fonológico típico e ao transtorno fonológico. Rev Soc Bras Fonoaudiol. 2004; 9(4):199-203.

\section{RECEBIDO EM: 23/11/06 \\ ACEITO EM:21/02/07}

Endereço para correspondência:

Rua Felipe Henn, 264

Três de Maio - RS

CEP: $98910-000$

Tel: (55) 35354112 / 99998945

E-mail: nubefono@yahoo.com.br
11. Grunwell P. Os desvios fonológicos evolutivos numa perspectiva lingüística. In: Yavas MS. Desvios fonológicos em crianças: teoria, pesquisa e tratamento. Porto Alegre: Mercado Aberto; 1990. p. 53-77.

12. Montenegro ACA, Costa TLS. Desvio fonético $x$ desvio fonológico: algumas considerações. J Bras Fonoaudiol. 2004; 5(21):258-63.

13. Vieira MG, Mota HB, Keske-Soares M. Relação entre idade, grau de severidade do desvio fonológico e consciência fonológica. Rev Soc Bras Fonoaudiol. 2004; 9(3):144-50.

14. Linassi LZ, Keske-Soares M, Mota HB. Habilidades de memória de trabalho e o grau de severidade do desvio fonológico. Pró-Fono R Atual Cient. 2005; 17(3):383-92.

15. Mota HB. Fonologia: intervenção. In: Ferreira LP, Befi-Lopes DM, Limongi SCO. Tratado de fonoaudiologia. São Paulo: Roca; 2004. p. 787-814. 16. Gierut JA. Maximal opposition approach to phonological treatment. J Speech Hear Disord. 1989; 54(1):9-19.

17. Gierut JA. The conditions and course of clinically induced phonological change. J Speech Hear Res. 1992; 35(5):1049-63.

18. Gierut JA, Simmerman CL, Neumann HJ. Phonetic structures of delayed phonological systems. J Child Lang. 1994; $21(2): 291-396$.

19. Pereira LF. Tratamento fonológico baseado nos contrastes de oposições máximas [mestrado]. Santa Maria (RS): Universidade Federal de Santa Maria; 1999.

20. Silva KC, Ramos APF, Wippel MLM. O modelo de oposições máximas como instrumental terapêutico em três casos de desvios fonológicos. J Bras Fonoaudiol. 2002; 3(13):292-7.

21. Bagetti T, Mota HB, Keske-Soares M. Modelo de oposições máximas modificado: uma proposta de tratamento para o desvio fonológico. Rev Soc Bras Fonoaudiol. 2005; 10(1):36-42.

22. Yavas M, Hernandorena C, Lamprecht R. Avaliação fonológica da criança. Porto Alegre: Artes Médicas; $1991.148 \mathrm{p}$.

23. Marchesan IQ. Fundamentos em fonoaudiologia: aspectos clínicos da motricidade oral. Rio de Janeiro: Guanabara Koogan; 1998. 108 p.

24. Capovilla AGS, Capovilla FC. Prova de consciência fonológica: desenvolvimento de dez habilidades da pré-escola à segunda série. Temas Desenvolv. 1998; 7(37):14-20.

25. Pereira LD. Avaliação do processamento auditivo central. In: Lopes Filho O. Tratado de fonoaudiologia. 1. ed. São Paulo: Roca; 1997. p. 109-26.

26. Mota HB, Bagetti T, Keske-Soares M, Pereira LF. A generalização em sujeitos com desvio fonológico médio-moderado tratados pelo modelo de oposições máximas. Rev Soc Bras Fonoaudiol. 2004; 9(2):102-11. 